\title{
Biomarkers in Heart Failure
}

Heart failure (HF), the end stage of all diseases of the heart is a major and growing public health problem particularly in elderly population. It appears to result not only from cardiac overload or injury but also from a complex interplay among genetic, neurohormonal, inflammatory and biochemical changes acting on cardiac myocytes, the cardiac interstitium or both.

Incidence of HF has been steadily increasing over the past several decades. The overall prevalence of HF is 3-20 per 1000 population with the annual incidence of HF 1-5 per 1000. The relative incidence doubles for each decade of life after the age of $45 \mathrm{yrs}$. The overall incidence is likely to increase in the future because of both an ageing population, a blessing of improved health care facilities and therapeutic advances in the management of acute MI leading to improved survival in patients with impaired cardiac function. ${ }^{1}$

Unfortunately, HF can be difficult to diagnose clinically, as many features of the condition are not organ specific and there may be few clinical features in the early stages of the disease. Recent exciting seminal advances in the management of HF have made the early recognition of $\mathrm{HF}$ an increasingly important clinical issue. So interest has intensified in developing biological markers to predict susceptibility and aid in the early diagnosis and management of HF.

\section{What is biomarker?}

Generally a biomarker is defined as measurable event in a biological system (e.g. human body) or, alternatively, a molecule that indicates alterations in physiology from normal. ${ }^{2}$

What constitutes an ideal biomarker in $\mathbf{H F}^{3,4}$

A biomarker to be useful clinically in the early diagnosis and management of HF must fulfill following important set criterias:

1. Highly sensitive and specific for $\mathrm{HF}$

2. Assay should be relatively easy to perform and accurate, repeated measurements must be available to the clinician at a reasonable cost and with short turnaround times.
3. Biological validity: changes in the level of the biomarker would accurately reflect the changes in the patient's clinical status. That is optimizing the level of biomarker through changes should translate into meaningful clinical outcomes and decision making.

4. Biomarker must provide information that is not already available from a careful clinical assessment.

Although relatively few of the biomarkers so far identified satisfy all these criterias, many appear to provide important information regarding the pathogenesis of HF or the identification of subjects at risk of HF or appear to be useful in risk stratification, in the diagnosis of HF, or in monitoring therapy.

\section{Biomarkers in $\mathrm{HF}$}

Although no specific classes for biomarkers are accepted, Braunwald ${ }^{5}$ proposed that they could be divided into six categories as follows according to the pathophysiological event of HF they committed to recognize.

\section{Inflammation}

Inflammation is important in the pathogenesis \& prognosis of many forms of HF. So biomarkers (inflammatory mediators) of inflammation have become the subject of inquiry.

e.g. CRP, TNF- $\alpha$, IL-1, 6,18

\section{Oxidative stress}

Increased oxidative stress results from an imbalance between generated reactive oxygen species (ROS) and endogenous antioxidant defense mechanisms. This imbalance exerts deleterious effects on endothelial function as well as on the pathogenesis \& prognosis of HF. e.g. oxidized LDL, myeloperoxidase, malondialdehyde, urinary biopyrines.

3. Cardiac extracellular matrix remodeling Remodeling of ventricles plays an important role in the progression of HF. The extracellular matrix provides a "skeleton" for myocytes and determines their size and shape. Normally there is a balance between matrix metalloproteinases (proteolytic 
enzymes that degrade fibrillar collagen) and tissue inhibitors of metalloproteinases. Dominance of matrix metalloproteinases cause ventricular dilatation \& remodeling.

e.g. matrix metalloproteinases, collagen peptides, metalloproteinase inhibitors.

\section{Neurohormones}

Sympathetic nervous system (SNS) and Reninangiotensine-aldosterone system (RAAS) gets activated \& contribute in pathogenesis of HF. e.g. plasma norepinephrine (PNE), Endothelin-1 (ET), Renin, Angiotensin -II.

\section{Myocyte stress}

e.g. Brain naturetic peptide (BNP), N- terminal pro-BNP (NT-Pro-BNP),

Atrial naturetic peptide (ANP), Adrenomedullin.

\section{Myocyte injury (due to ischemia or stress)}

e.g. Cardiac specific troponin-I (cTn-I), Cardiac specific troponin-T (cTn-T),

CPK-MB, Heart-type fatty acid binding protein, Myosin light-chain kinase-1

\section{Biomarkers seems to be promising at present}

Although HF literature is rife with examples of circulating biomarkers that predict poor outcomes in patients with $\mathrm{HF}$, there is far less information regarding the use of biomarkers to predict changes in the clinical status of patient. Of the myriad of biomarkers that have been examined, BNP and PNE are the best characterized biomarkers that have been studied in the context of HF. ${ }^{3}$ Anand and Colleagues ${ }^{6}$ observed that the relative risk of having an event was significantly higher for those HF patients in whom the baseline BNP \& PNE levels were significantly elevated. The authors also found that BNP was more sensitive predictor of morbidity \& mortality than was PNE. However the salient finding in this study was that changes in BNP and PNE levels tracked therapeutic outcome. That is morbidity and mortality were least in those patients with greatest decrease in BNP \& PNE levels, whereas the morbidity \& mortality were greatest in those patients with the greatest percentage increase in BNP \& PNE levels during the course of the trial. Low circulating PNE and better clinical outcome in HF patients has also been documented by CONCENSUS ${ }^{7}$ and SOLVD $^{8}$ studies.

Plasma naturetic peptides (ANP, BNP) are increasingly being recognized as important prognostic markers in patients with HF. Nonetheless, the extant clinical literature suggests that ANP levels are less reliable in terms of predicting clinical outcomes in HF than are BNP levels. ${ }^{3}$ Several smaller single-center studies have confirmed the utility of BNP measurements in assessing clinical outcomes in HF \& these studies finally concluded that low plasma BNP at the outset can predict better outcomes. 9,10 Precursor of BNP is a preprohormone (134AA) synthesized in myocytes and cleaved to the prohormone (108AA) which is released in blood during hemodynamic stress (when ventricle wall tension increase e.g. HF). On coming to blood prohormone is cleaved by a circulating endoprotease (called corin) into bioactive BNP (32AA) \& an inactive NT-pro-BNP (76AA). ${ }^{11}$ The more the ventricular wall stress (as in progressive $\mathrm{HF}$ ) the more will be the plasma BNP level. So BNP deserved to be used as a diagnostic \& prognostic biomarker in $\mathrm{HF}$ and also as a tool for risk stratification of patients with HF. ${ }^{12}$ Hammerer-Lercher ${ }^{13}$ evaluated the diagnostic performance of BNP in patients with left ventricular dysfunction and showed sensitivity and specificity $73 \%$ \& $77 \%$ respectively. BNP has also been shown to be a marker of diastolic dysfunction \& in this regard taking the cut off point of $62 \mathrm{ng} / \mathrm{L}$ the sensitivity, specificity and accuracy of BNP for detecting diastolic dysfunction found to be $85 \%$, $83 \%$ and $84 \%$ respectively. ${ }^{14}$ Utility of BNP in the emergency diagnosis of HF with preserved ejection fraction was evaluated in large study involving patients with acute dyspnea and its sensitivity, negative predictive value \& accuracy found to be $86 \%, 96 \%$ \& $75 \%$ respectively to diagnose diastolic dysfunction at a cut off point of BNP $100 \mathrm{ng} / \mathrm{L} .{ }^{15}$ BNP also appear to be useful in screening asymptomatic subjects at risk of developing HF (e.g. elderly and those with HTN, DM). ${ }^{11}$ Hobbs $^{16}$ advocate that, rapid assay of BNP not only can be used to diagnose HF, it can help the clinician evaluate effectiveness of therapy, determine when discharge from the hospital is appropriate and estimate prognosis. He suggested the plasma BNP concentration in health and disease as follows.

\begin{tabular}{cc}
\hline $\begin{array}{c}\text { BNP level } \\
(\mathbf{p g} / \mathbf{m l} \text { or } \mathbf{n g} / \mathbf{L})\end{array}$ & $\begin{array}{c}\text { Clinical } \\
\text { condition }\end{array}$ \\
\hline$<100$ & Normal \\
$<500$ & Goal at hospital discharge \\
$\geq 700$ & Decompensated CCF \\
\hline
\end{tabular}




\section{Future directions \& Conclusion:}

Biomarkers have been present in cardiology for many years. They have expanded their role from merely being adjunctive to become the gold diagnostic standard and strong prognosticators in a variety of cardiac conditions. HF is no exception to this \& has a long \& exciting history of biomarkers. Naturetic peptides moved the field forward in the last 20 years but we have not yet achieved the level of sophistication that is necessary to reliably use biomarkers to optimize clinical care for patients with HF. Nevertheless a biomarker profile may be a valuable addition to the traditional approach to the patients of HF with respect to diagnosis, therapy \& prognosis. A multimarker strategy has been reported to be more useful, so one might envision that clinicians could use along with clinical parameters, combination of different biomarkers that reflect different aspects of the disease process to optimize facets of patient care. For example, the use of data on BNP together with troponin has been shown to achieve better risk stratification than that obtained with either biomarker alone. The accuracy of risk prediction was enhanced when BNP was coupled with other biomarkers like adrenomedullin, CRP, IL, myeloperoxidase etc. Although it is always difficult to make accurate predictions about future therapeutic approaches in HF, the wealth of emerging clinical data suggests that it is increasingly likely that clinicians will one day have sufficient information to use biomarkers to facilitate and optimizes the care for their HF patient.

Please remember "what gets you into trouble is not what you don't know, it's what you know for sure, that just ain't so".

(J Bangladesh Coll Phys Surg 2008; 26: 112-114)

\section{Prof. Md. Mozammel Hoque \\ Dept. Biochemistry \\ Bangabandhu Sheikh Mujib Medical University, Dhaka}

\section{References:}

1. Davis RC, Hobbs FDR, LipGYH. ABC of heart failure History and epidemiology. BMJ 2000; 320: 39-42.

2. State EH, Turnbull BW. Statistical models of longitudinal biomarkers of disease. Stat Med 2000; 19: 617-37.
3. Bozkurt B, Mann DL. Use of biomarkers in the management of heart failure: are we there yet? Circulation 2003; 1231-1233.

4. Morrow DA, de Lemos JA. Benchmarks for the assessment of novel cardiovascular markers. Circulation 2007; 115: 949-52.

5. Braunwald E. Biomarkers in heart failure. $\mathrm{N}$ engl J Med 2008; 358; 20: 2148-2159.

6. Anand IS, Fisher LD, Chiang Y-T. et al. Changes in brai naturetic peptide and norepinephrine over time and mortality and morbidity in Val-HeFT. Circulation 2003; 107: $1278-1283$.

7. Swedberg K, Eneroth P, Kjekshus J. et al. Hormones regulating cardiovascular function in patients with severe congestive heartfailure and their relation to mortality. Circulation 1990; 82: 1730-1736.

8. BenedictCR, Francis GS, Shelton B. et al. Effect of long term enalapril therapy on Neurohormones in patients with left ventricular dysfunction. Am J Cardiol 1995; 75: 1151-1157.

9. Richards AM, Doughty R, Nicholls MG. et al. Neurohumoral prediction of benefit from carvedilol in ischemic left ventricular dysfunction. Australia-New Zealand Heart Failure Group. Circulation 1999; 99: 786-792.

10. Tsutamoto T, Wada A, Maeda K, et al. Effect of spironolactone on plasma brain naturetic peptide and left ventricular remodeling in patients with congestive heart failure. J Am Coll Cardiol 2001; 37(5): 1228-1233.

11. Daniels LB, Maisel AS. Naturetic peptides. J am Coll Cardiol. 2007; 50: 2357-68.

12. Sugiura T, Takase H, Toriyama T, Goto T, Ueda R, Dohi Y. Circulating levels of myocardial proteins predict future deterioration of congestive heart failure. J Card Fail 2005; 11: 504-9.

13. Hammwerer-Lercher A, Neubauer E, Muller S, Pachinger O, Puschendrof B, Mair J, Head to head comparison of Nterminal pro-brain naturetic peptide, brain naturetic peptide nad N-terminal pro-trial naturetic peptide in diagnosing left ventricular dysfunction. Clin Chim Acta 2001; 310: 193-27.

14. Lubien E, DeMasria A, Krishnaswamy P, Clopton P, Koon J. Kazanegra R, etal. Utility of B-naturetic peptide in detecting diastolic dysfunction: comparison with Doppler velocity recordings. Circulation 2002; 105: 595-601.

15. Maisel AS, McCord J, Nowak RM, Hollander JE, WU AH, Duc $\mathrm{P}$, et al. Bedside B type naturetic peptide in emergency diagnosis of heart failure with reduced or preserved ejection fraction. Results from Breathing Not Properly Multinational Study. J AM Coll Cardio 2003; 41: 2010-7.

16. Hobbs RE. Using of BNP to diagnose, manage and treat heart failure. Cleveland Clinic J Med 2003; 70: 333-33s6 